\title{
Christensen measurability and some functional equation
}

\author{
ELIZA JABŁOŃSKA
}

\begin{abstract}
Let $X$ be a real separable $F$-space. We characterize solutions $f: X \rightarrow \mathbb{R}$ and $M: \mathbb{R} \rightarrow \mathbb{R}$ of the equation $f(x+M(f(x)) y)=f(x) f(y)$ such that $f$ is bounded on a nonzero Christensen measurable set. Our result generalizes [Jabłońska in Acta Math Hung 125(1-2):113-119 2009, Theorem 1].
\end{abstract}

Mathematics Subject Classification (2000). Primary 39B52, Secondary 28E05.

Keywords. Christensen measurability, Gołąb-Schinzel equation, exponential function, bounded solutions.

\section{Introduction}

Let $\mathbb{N}, \mathbb{R}, \mathbb{C}$ denote the sets of positive integers, reals and complex numbers, respectively. We consider the equation

$$
f(x+M(f(x)) y)=f(x) f(y),
$$

where $f: X \rightarrow \mathbb{R}$ mapping a real separable $F$-space $X$ into $\mathbb{R}$ and $M: \mathbb{R} \rightarrow \mathbb{R}$ are unknown functions. This equation generalizes two classical functional equations: the exponential equation

$$
f(x+y)=f(x) f(y)
$$

and the Gołąb-Schinzel equation

$$
f(x+f(x) y)=f(x) f(y)
$$

(for $M=1$ and $M=\mathrm{id}_{\mathbb{R}}$, respectively). Equation (2) is very well known; for more information on it we refer to [1, pp. 25-33, 52-57]. The Gołąb-Schinzel equation was introduced in [16] by S. Gołąb and A. Schinzel in connection with the determination of subgroups of the centroaffine group of the plane. It turned out that this equation also has applications in the determination of substructures of algebraic structures, in the theory of geometric objects, in theories 
of near-rings and quasialgebras as well as in meteorology and fluid mechanics. That is why this equation and its generalizations have been considered by many authors in various classes of functions (for more information on (3), its applications and generalizations we refer to a survey paper [6]). In recent years stability of equations of the Gołąb-Schinzel type has been extensively studied (see [7], [10]-[14]).

It seems to be well known that each of the positive Lebesgue measure solution $f: \mathbb{R}^{k} \rightarrow \mathbb{R}$ (for $k \in \mathbb{N}$ ) of Eq. (2) that is bounded on a set is continuous. This result is a consequence of a theorem of A. Ostrowski (see e.g. [1, Theorem 5 , p. 29]).

For the first time solutions of an equation of the Gołąb-Schinzel type bounded on a "big" set were studied by J. Brzdȩk [5]. He proved that each function $f$ mapping a separable $F$-space $X$ over $\mathbb{K} \in\{\mathbb{R}, \mathbb{C}\}$ into $\mathbb{K}$ satisfying the equation

$$
f\left(x+f(x)^{n} y\right)=f(x) f(y) \quad \text { with some } \quad n \in \mathbb{N}
$$

and such that $|f(x)| \subset(0, a)$ for every $x \in A$, for a set $A \subset X$ of second category with the Baire property and for some $a>0$, is bounded or continuous and, if $\mathbb{K}=\mathbb{R}$, then $f$ has to be continuous. An analogous result for solutions of (4) that are bounded on nonzero Christensen measurable sets was proved in [20] (see also [18]). Moreover, in [23] solutions of (1) bounded on "big" sets in some abstract sense were studied.

In this paper we characterize solutions $f: X \rightarrow \mathbb{R}$ mapping a real separable $F$-space $X$ into $\mathbb{R}$ and $M: \mathbb{R} \rightarrow \mathbb{R}$ of (1) under the assumption that $|f(x)| \subset(0, a)$ for every $x \in A$, for some nonzero Christensen measurable set $A \subset X$ and for some $a>0$. Our result refers to the question of J. Brzdȩk $[6$, Problem, p. 18].

Through the paper $m, m_{e}$ and $m_{i}$ denote the Lebesgue, the outer Lebesgue and the inner Lebesgue measure in $\mathbb{R}$, respectively. Next, for a real linear space $X, a \in \mathbb{R}, V, W \subset \mathbb{R}, x_{0} \in X$ and $A, B \subset X$ :

$\operatorname{lin} A$ denotes the linear subspace of $X$ spanned by $A$ over $\mathbb{R}$,

$$
\begin{aligned}
& A+B:=\{x+y: x \in A, y \in B\}, \\
& A+x_{0}:=A+\left\{x_{0}\right\}, \\
& V A:=\{b x: b \in V, x \in A\}, \\
& V x_{0}:=V\left\{x_{0}\right\}, \\
& W \cdot V:=\{a b: a \in W, b \in V\}, \\
& |V|:=\{|a|: a \in V\} .
\end{aligned}
$$

Moreover, for a function $f: X \rightarrow \mathbb{R}$ mapping a real linear space $X$ into $\mathbb{R}$ and an $x \in X \backslash\{0\}$, we define sets:

$$
A_{f}:=f^{-1}(\{1\}), \quad F_{f}:=\{x \in X: f(x) \neq 0\}, \quad W_{f}:=f(X) \backslash\{0\}=f\left(F_{f}\right)
$$


and a function $f_{x}: \mathbb{R} \rightarrow \mathbb{R}$,

$$
f_{x}(a)=f(a x) \quad \text { for each } a \in \mathbb{R} .
$$

\section{Preliminary lemmas}

First we recall some lemmas which will be useful in what follows.

Lemma 1 ([3, Lemma 2]). Let $B$ be a subgroup of $(X,+)$ and let $V$ be an infinite subgroup of $(\mathbb{R} \backslash\{0\}, \cdot)$ such that $V B \subset B$. Then, for every $x \in B$, the set $B_{x}=\{a \in \mathbb{R}: a x \in B\}$ is dense in $\mathbb{R}$.

In the next lemmas we recall some general properties of functions satisfying (1).

Lemma 2 ([17, Lemma $\mathbf{2}$, Lemma 3$])$. Let $X$ be a real linear space, $f: X \rightarrow \mathbb{R}$, $f \neq 0, f \neq 1$ and $M: \mathbb{R} \rightarrow \mathbb{R}$. If $f$ and $M$ satisfy (1), then:

(i) $f\left(M(f(x))^{-1}(z-x)\right)=f(z) f(x)^{-1}$ for $x \in F_{f}$ and $z \in X$;

(ii) $(M \circ f)^{-1}(\{0\})=f^{-1}(\{0\})$;

(iii) $M\left(W_{f}\right) A_{f} \subset A_{f}$;

(iv) $A_{f}$ is a subgroup of $(X,+)$;

(v) $A_{f} \backslash\{0\}$ is the set of periods of $f$ (i.e. $f(x+z)=f(x)$ for every $x \in X$ and $\left.z \in A_{f} \backslash\{0\}\right)$;

(vi) $W_{f}$ is a subgroup of $(\mathbb{R} \backslash\{0\}, \cdot)$;

(vii) $x-y \in A_{f}$ for every $x, y \in F_{f}$ with $f(x)=f(y)$.

Lemma 3 ([19, Lemma 3$])$. Let $X$ be a real linear space, $f: X \rightarrow \mathbb{R}, f \neq 0$, $f \neq 1$ and $M: \mathbb{R} \rightarrow \mathbb{R}$. If $f$ and $M$ satisfy (1), then:

(i) there exists a function $w: W_{f} \rightarrow X$ such that $F_{f}=w\left(W_{f}\right)+A_{f}$;

(ii) $f$ and $\widetilde{M}: \mathbb{R} \rightarrow \mathbb{R}$ satisfy (1), where

$$
\widetilde{M}(a)=\frac{M(a)}{M(1)} \quad \text { for each } \quad a \in \mathbb{R}
$$

(iii) if, moreover, $M(1)=1$ and $M \circ f \neq 1$, then $0 \in f(X)$.

Lemma 4 ([17, Lemma 5, Lemma 6]). Let $X$ be a real linear topological space, $f: X \rightarrow \mathbb{R}, M: \mathbb{R} \rightarrow \mathbb{R}, M(1)=1$ and $M \circ f \neq 1$. If $f$ and $M$ satisfy (1) and $0 \in \operatorname{int} F_{f}$, then $f(X)$ is infinite and $M\left(W_{f}\right) \backslash\{-1,1\} \neq \emptyset$.

Lemma 5 ([19, Lemma 4]). Let $X$ be a real linear space, $f: X \rightarrow \mathbb{R}, M: \mathbb{R} \rightarrow$ $\mathbb{R}, f \neq 0, M(1)=1$ and $M\left(W_{f}\right) \backslash\{1\} \neq \emptyset$. Let functions $f$ and $M$ satisfy (1). Then there exists $x_{0} \in X$ such that

$$
F_{f} \subset\left(M\left(W_{f}\right)-1\right) x_{0}+\operatorname{lin} A_{f} .
$$


Furthermore, if $A_{f}=\operatorname{lin} A_{f}$, then $x_{0} \notin A_{f}$,

$$
f^{-1}(\{f(x)\})=(M(f(x))-1) x_{0}+A_{f} \quad \text { for each } \quad x \in F_{f}
$$

and $\left.M\right|_{f(X)}$ is injective and multiplicative.

\section{Christensen measurability}

We start with some basic definitions which can be found in [8]-[9] and [15].

Let $X$ be a real separable $F$-space and let $\mathfrak{M}$ be the $\sigma$-algebra of all the universally measurable subsets of $X$; i.e. $\mathfrak{M}$ is the intersection of all the completions of the Borel $\sigma$-algebra of $X$ with respect to probability Borel measures. In the following a measure is a countable additive Borel measure extended to $\mathfrak{M}$.

Definition 1. A set $B \in \mathfrak{M}$ is a Haar zero set iff there exists a probability measure $u$ on $X$ such that $u(B+x)=0$ for each $x \in X$. A set $P \subset X$ is a Christensen zero set iff $P$ is a subset of a Haar zero set. A set $D \subset X$ is a Christensen measurable set iff there are $B \in \mathfrak{M}$ and a Christensen zero set $P$ such that $D=B \cup P$. A function $f: X \rightarrow \mathbb{R}$ is said to be Christensen measurable iff $f^{-1}(U)$ is Christensen measurable for each open set $U \subset \mathbb{R}$.

Denote

$$
\begin{gathered}
\mathcal{C}_{0}:=\{B \subset X: B \text { is Christensen zero set }\}, \\
\mathcal{C}:=\{B \subset X: B \text { is Christensen measurable }\} .
\end{gathered}
$$

The family $\mathcal{C}_{0}$ is a $\sigma$-ideal and the family $\mathcal{C}$ is a $\sigma$-algebra (see $[8$, Theorem 1$]$ and [15, Proposition 1]).

Lemma 6 ([8, Theorem 2]). If $B \in \mathcal{C} \backslash \mathcal{C}_{0}$, then $0 \in \operatorname{int}(B-B)$.

Lemma 7 ([4, Lemma 14]). Let $D \in \mathcal{C} \backslash \mathcal{C}_{0}$ and $x \in X \backslash\{0\}$. Then there exist a Borel set $D_{x} \subset D$ and $y_{x} \in X$ such that

$$
m\left(k_{x}^{-1}\left(y_{x}+D_{x}\right)\right)>0,
$$

where $k_{x}: \mathbb{R} \rightarrow X, k_{x}(a)=a x$.

Lemma 8 ([21, Corollary $])$. Each additive function $a: X \rightarrow \mathbb{R}$ bounded above on a nonzero Christensen measurable set is linear.

Lemma 9 ([22, Lemma 13]). Let $f: X \rightarrow \mathbb{R}, f \neq 1, M: \mathbb{R} \rightarrow \mathbb{R}$ and let $D \in \mathcal{C} \backslash \mathcal{C}_{0}$ be such that $D \subset F_{f}$. If $f$ and $M$ satisfy (1), then, for each $x \in X \backslash\{0\}$, there is a $z \in F_{f}$ with $m_{i}\left(f_{x}^{-1}\left(f(z)^{-1} f(D)\right)\right)>0$.

Now, we prove two lemmas. 
Lemma 10. If functions $f: X \rightarrow \mathbb{R}$ and $M: \mathbb{R} \rightarrow \mathbb{R}$ satisfy (1), $M(1)=1$, $M \circ f \neq 1$ and there is a set $D \in \mathcal{C} \backslash \mathcal{C}_{0}$ such that $D \subset F_{f}$, then $f(X)$ is infinite and $M\left(W_{f}\right) \backslash\{-1,1\} \neq \emptyset$.

Proof. First suppose that $M\left(W_{f}\right)=\{1\}$. Then, in view of (1),

$$
f(x+y)=f(x) f(y) \neq 0 \quad \text { for every } \quad x, y \in F_{f}
$$

and thus $F_{f}+F_{f} \subset F_{f}$. Applying Lemma 2(i) with $z=0$ we obtain $F_{f}=-F_{f}$. Hence, by Lemma 6,

$$
0 \in \operatorname{int}(D-D) \subset \operatorname{int}\left(F_{f}-F_{f}\right) \subset \operatorname{int} F_{f}
$$

and, on account of Lemma $4, M\left(W_{f}\right) \backslash\{-1,1\} \neq \emptyset$, which is a contradiction. Thus $M\left(W_{f}\right) \backslash\{1\} \neq \emptyset$.

Next, suppose that $M\left(W_{f}\right)=\{-1,1\}$. Fix an $x \in F_{f}$. Then either $M(f(x))=-1$, or $M(f(x))=1$. In the first case, by (1) and Lemma 2(iv), we trivially get $f(x)^{2}=1$. Since $M(1)=1$, we have $f(x)=-1$. In the second one, taking a $y \in F_{f}$ with $M(f(y))=-1$ (since $M\left(W_{f}\right)=\{-1,1\}$ such a $y$ exists), in view of (1), we obtain

$$
f(y-x)=f(y+M(f(y)) x)=f(x) f(y)=f(x+M(f(x)) y)=f(x+y) .
$$

Hence, applying Lemma 2(vii), we get $f(2 x)=1$ and so, by (1),

$$
f(x)^{2}=f(x+M(f(x)) x)=f(2 x)=1 .
$$

Now, using Lemma 2(vi), we obtain that $W_{f}=\{-1,1\}$.

Since $M \circ f \neq 1$ and $M(1)=1, f \neq 1$. Fix $z \in X \backslash A_{f}$. By Lemma 9 there is a $z_{0} \in F_{f}$ with $m_{i}\left(f_{z}^{-1}\left(f\left(z_{0}\right)^{-1} f(D)\right)\right)>0$. Condition $D \subset F_{f}$ implies $f\left(z_{0}\right)^{-1} f(D) \subset \mathbb{R} \backslash\{0\}$. Hence $f_{z}^{-1}\left(f\left(z_{0}\right)^{-1} f(D)\right) \subset f_{z}^{-1}(\mathbb{R} \backslash\{0\})$ and, consequently, $m_{i}\left(f_{z}^{-1}(\mathbb{R} \backslash\{0\})\right)>0$. Clearly $f_{z}$ and $M$ satisfy $(1), f_{z} \neq 0$ and $f_{z} \neq 1$. Then, according to Lemma $3(\mathrm{i})$, there is a function $w: W_{f_{z}} \rightarrow \mathbb{R}$ such that

$$
F_{f_{z}}=\bigcup_{a \in W_{f_{z}}}\left(w(a)+A_{f_{z}}\right) .
$$

Since $W_{f_{z}} \subset W_{f}=\{-1,1\}$ and $m_{i}\left(F_{f_{z}}\right)>0$, we have $m_{e}\left(A_{f_{z}}\right)>0$. In view of Lemma 2(v) $F_{f_{z}}+A_{f_{z}} \subset F_{f_{z}}$. Now, by [2, Theorem 1], $\emptyset \neq \operatorname{int}\left(F_{f_{z}}+A_{f_{z}}\right) \subset$ $\operatorname{int} F_{f_{z}}$ and hence, using Lemma $4, W_{f_{z}}$ is infinite. This contradiction ends the proof.

An idea of the proof of the next lemma is similar to the idea of the proof of Lemma 9 in [20]. Therefore, some parts of the proof are analogous to those in $[20]$.

Lemma 11. Let functions $f: X \rightarrow \mathbb{R}$ and $M: \mathbb{R} \rightarrow \mathbb{R}$ satisfy (1), $M \circ f \neq 1$ and $M(1)=1$. If there is a set $D \in \mathcal{C} \backslash \mathcal{C}_{0}$ such that $|f(D)| \subset(0, a)$ for $a$ positive real a, then $\operatorname{lin} A_{f} \neq X$. 
Proof. For an indirect proof suppose that $\operatorname{lin} A_{f}=X$. In the first step we prove that there are $y \in A_{f} \backslash\{0\}$ and $a_{0} \in \mathbb{R}$ fulfilling $f\left(a_{0} y\right)=0$. To this end first consider the case where

$$
\left.M \circ f\right|_{\mathbb{R} y}=1 \text { for each } y \in A_{f} \backslash\{0\} .
$$

Since $\operatorname{lin} A_{f}=X, A_{f}$ must contain a base for $X$. Thus, by Lemma 3(iii), there are $\alpha_{i} \in \mathbb{R}$ and $z_{i} \in A_{f}$, where $i \in\{1, \ldots n\}$ for some $n \in \mathbb{N}$, such that $f\left(\sum_{i=1}^{n} \alpha_{i} z_{i}\right)=0$. Moreover, $(M \circ f)\left(\alpha_{i} z_{i}\right)=1$ for each $i \in\{1, \ldots, n\}$. Hence, in view of (1), using induction we obtain that

$$
\begin{aligned}
0 & =f\left(\sum_{i=1}^{n} \alpha_{i} z_{i}\right)=f\left(\alpha_{1} z_{1}+M\left(f\left(\alpha_{1} z_{1}\right)\right)\left(\sum_{i=2}^{n} \alpha_{i} z_{i}\right)\right) \\
& =f\left(\alpha_{1} z_{1}\right) f\left(\sum_{i=2}^{n} \alpha_{i} z_{i}\right)=f\left(\alpha_{1} z_{1}\right) f\left(\alpha_{2} z_{2}+M\left(f\left(\alpha_{2} z_{2}\right)\right)\left(\sum_{i=3}^{n} \alpha_{i} z_{i}\right)\right) \\
& =f\left(\alpha_{1} z_{1}\right) f\left(\alpha_{2} z_{2}\right) f\left(\sum_{i=3}^{n} \alpha_{i} z_{i}\right)=\cdots=\prod_{i=1}^{n} f\left(\alpha_{i} z_{i}\right) .
\end{aligned}
$$

Consequently, $f\left(\alpha_{j} z_{j}\right)=0$ for some $j \in\{1, \ldots, n\}$. In the case where

$$
\left.M \circ f\right|_{\mathbb{R} y} \neq 1 \quad \text { for some } y \in A_{f} \backslash\{0\},
$$

functions $\left.f\right|_{\mathbb{R} y}$ and $M$ satisfy (1) and hence, by Lemma 3(iii), $f\left(a_{0} y\right)=0$ for some $a_{0} \in \mathbb{R}$.

Now, fix $y \in A_{f} \backslash\{0\}$ such that $f\left(a_{0} y\right)=0$ for some $a_{0} \in \mathbb{R}$. Clearly then $f_{y}$ and $M$ satisfy $(1), f_{y} \neq 1, f_{y} \neq 0$ (because $f_{y}\left(a_{0}\right)=0$ and $f_{y}(1)=1$ ) and, by Lemma 2(ii), $M \circ f_{y} \neq 1$. According to Lemma 2(iv), $A_{f}$ is a subgroup of the group $(X,+)$. Simultaneously, by Lemma 10, the multiplicative group $\left\langle M\left(W_{f}\right)\right\rangle$ generated by $M\left(W_{f}\right)$ is infinite and, in view of Lemma 2(iii), $\left\langle M\left(W_{f}\right)\right\rangle A_{f} \subset A_{f}$. Hence, using Lemma $1, A_{f_{y}}=\left\{a \in \mathbb{R}: a y \in A_{f}\right\}$ is dense in $\mathbb{R}$. Moreover, from Lemma 9 we obtain that there is some $z \in F_{f}$ with $m_{i}\left(f_{y}^{-1}\left(f(z)^{-1} f(D)\right)\right)>0$. Let $B \subset f_{y}^{-1}\left(f(z)^{-1} f(D)\right)$ be such that $m(B)>0$. Since $|f(D)| \subset(0, a),\left|f_{y}(B)\right| \subset\left|f(z)^{-1} f(D)\right| \subset\left(0, a_{0}\right)$ for $a_{0}=|f(z)|^{-1} a$. Hence, in view of Lemma 10, the set $W_{f_{y}}$ is infinite. Define a set

$$
D_{0}=\left\{c \in F_{f_{y}}:|f(c)|<a_{0}\right\} .
$$

Clearly $B \subset D_{0}$. Moreover, by Lemma $2(\mathrm{v}), A_{f_{y}}+D_{0} \subset D_{0}$. Hence we obtain

$$
A_{f_{y}} \cap\left[\left(\mathbb{R} \backslash D_{0}\right)-D_{0}\right]=\emptyset .
$$

Thus $m_{e}\left(\mathbb{R} \backslash D_{0}\right)=0$, because otherwise, in view of $[2$, Theorem 1], we would have

$$
\operatorname{int}\left(\left(\mathbb{R} \backslash D_{0}\right)-D_{0}\right) \supset \operatorname{int}\left(\left(\mathbb{R} \backslash D_{0}\right)-B\right) \neq \emptyset,
$$


which contradicts the density of $A_{f_{y}}$. Now, according to Lemma 2(vi), there is a sequence $\left(b_{m}\right)_{m \in \mathbb{N}} \subset F_{f_{y}}$ such that for each $b \in F_{f_{y}}$ there exists some $m \in \mathbb{N}$ with

$$
f_{y}\left(b_{m}+M\left(f\left(b_{m}\right)\right) b\right)=f_{y}\left(b_{m}\right) f_{y}(b) \in f_{y}\left(\mathbb{R} \backslash D_{0}\right) .
$$

By Lemma 2(i)

$$
B \subset F_{f_{y}} \subset \bigcup_{m \in \mathbb{N}}\left(\left(\mathbb{R} \backslash D_{0}\right)-b_{m}\right) M\left(f_{y}\left(b_{m}\right)\right)^{-1} .
$$

Since $m_{e}\left(\mathbb{R} \backslash D_{0}\right)=0, m_{e}(B)=0$. This contradiction ends the proof.

\section{The main result}

Now, we are in a position to prove the announced theorem. Some parts of the proof are similar to the proof of Theorem 1 in [20], but the whole proof is more complicated because of the two unknown functions.

Theorem 1. Let $f: X \rightarrow \mathbb{R}, M: \mathbb{R} \rightarrow \mathbb{R}$ and $|f(D)| \subset(0, a)$ for a set $D \in \mathcal{C} \backslash \mathcal{C}_{0}$ and a positive number $a$. Then functions $f$ and $M$ satisfy (1) if and only if one of the following conditions holds:

(i) $f=1$;

(ii) $\left.M\right|_{(0, \infty)}=1$ and there exists a nontrivial linear functional $h: X \rightarrow \mathbb{R}$ such that $f(x)=\exp h(x)$ for each $x \in X$;

(iii) there exist a nontrivial linear functional $h: X \rightarrow \mathbb{R}$ and $a c \in \mathbb{R} \backslash\{0\}$ such that either

$$
\begin{aligned}
M(y) & =|y|^{\frac{1}{c}} \operatorname{sgn} y \quad \text { for } y \in \mathbb{R}, \\
f(x) & = \begin{cases}|h(x)+1|^{c} \operatorname{sgn}(h(x)+1), & x \in X, h(x) \neq-1 ; \\
0, & x \in X, h(x)=-1\end{cases}
\end{aligned}
$$

or

$$
\begin{aligned}
M(y) & =y^{\frac{1}{c}} \quad \text { for } y \in[0, \infty), \\
f(x) & = \begin{cases}(h(x)+1)^{c}, & x \in X, h(x)>-1 \\
0, & x \in X,\end{cases}
\end{aligned}
$$

Proof. First assume that $f$ and $M$ satisfy (1) and there is a set $D \in \mathcal{C} \backslash \mathcal{C}_{0}$ such that $|f(D)| \subset(0, a)$ for some $a>0$. It is clear that condition (i) of this Theorem holds. So assume that $f \neq 1$.

If $M \circ f=c$, then $f$ with $\widetilde{M}$ given by (5) fulfill (1) and $\widetilde{M} \circ f=1$. Hence, in view of (1), $f$ satisfies (2). Since $f \neq$ const, by [1, Theorem 5, p. 29], there is an additive function $h: X \rightarrow \mathbb{R}, h \neq 0$, such that $f=\exp h$. Moreover, $|f(D)| \subset(0, a)$ implies $h(x) \leq \ln a$ for every $x \in D$. Hence, according to Lemma $8, h$ is linear. Then $f(X)=(0, \infty)$. Putting $x=0$ in $(1)$, we have 
$f((c-1) y)=1$ for each $y \in X$. Thus $c=1$ and condition (ii) of this Theorem holds.

Now, let $M \circ f \neq c$. Then $f$ with $\widetilde{M}$ given by (5) also satisfy (1), $\widetilde{M}(1)=1$ and $\widetilde{M} \circ f \neq 1$. By Lemma $10 \widetilde{M}\left(W_{f}\right) \backslash\{-1,1\} \neq \emptyset$. Hence, on account of Lemma 5 , there is some $x_{0} \in X$ such that

$$
F_{f}-F_{f} \subset\left(\widetilde{M}\left(W_{f}\right)-\widetilde{M}\left(W_{f}\right)\right) x_{0}+\operatorname{lin} A_{f} .
$$

In view of Lemma $6, \operatorname{int}\left(F_{f}-F_{f}\right) \supset \operatorname{int}(D-D) \neq \emptyset$ and, by Lemma 11, $\operatorname{lin} A_{f} \neq X$. Hence $x_{0} \notin \operatorname{lin} A_{f}$. Consequently we obtain

$$
\mathbb{R} x_{0}+\operatorname{lin} A_{f}=X
$$

and

$$
\operatorname{int}\left(\widetilde{M}\left(W_{f}\right)-\widetilde{M}\left(W_{f}\right)\right) \neq \emptyset .
$$

Clearly then $\operatorname{int}\left(\left\langle\widetilde{M}\left(W_{f}\right)\right\rangle-\left\langle\widetilde{M}\left(W_{f}\right)\right\rangle\right) \neq \emptyset$, where $\left\langle\widetilde{M}\left(W_{f}\right)\right\rangle$ denotes the subgroup of $(\mathbb{R} \backslash\{0\}, \cdot)$ generated by $\widetilde{M}\left(W_{f}\right)$. In view of Lemma 2(iii) $\left\langle W_{f}\right\rangle A_{f} \subset A_{f}$. Thus, using Lemma 2(iv), $\mathbb{R} A_{f} \subset A_{f}$ and hence $A_{f}=\operatorname{lin} A_{f}$. Then, on account of Lemma $5, x_{0} \notin A_{f}$,

$$
f^{-1}(\{f(x)\})=(M(f(x))-1) x_{0}+A_{f} \text { for each } x \in F_{f}
$$

and the function $\left.\widetilde{M}\right|_{f(X)}$ is injective and multiplicative. Thus, in view of Lemma 2(vi), we obtain that $\widetilde{M}\left(W_{f}\right)$ is a subgroup of the group $(\mathbb{R} \backslash\{0\}, \cdot)$.

It results from (9) that

$$
X=\mathbb{R} x_{0}+A_{f} .
$$

Consequently, according to Lemma 7 , there are a Borel set $B_{0} \subset F_{f}, a \in \mathbb{R}$ and $x \in A_{f}$ such that

$$
m\left(k_{0}^{-1}\left(a x_{0}+x+B_{0}\right)\right)>0,
$$

where $k_{0}: \mathbb{R} \rightarrow X, k_{0}(a)=a x_{0}$. By (10) we have

$$
F_{f}=\left(\widetilde{M}\left(W_{f}\right)-1\right) x_{0}+A_{f}
$$

and thus

$$
a x_{0}+x+F_{f}=\left(\widetilde{M}\left(W_{f}\right)-1+a\right) x_{0}+A_{f} .
$$

Hence

$$
k_{0}^{-1}\left(a x_{0}+x+B_{0}\right) \subset k_{0}^{-1}\left(a x_{0}+x+F_{f}\right)=\widetilde{M}\left(W_{f}\right)-1+a .
$$

It means that $m_{i}\left(\widetilde{M}\left(W_{f}\right)\right)>0$ and, in view of a consequence of Steinhaus' Theorem (see [4, Lemma 10]),

$$
\operatorname{int} \widetilde{M}\left(W_{f}\right) \supset \operatorname{int}\left[\widetilde{M}\left(W_{f}\right) \cdot \widetilde{M}\left(W_{f}\right)\right] \neq \emptyset .
$$


But $\widetilde{M}\left(W_{f}\right)$ is a subgroup of $(\mathbb{R}, \cdot)$. Hence

$$
\widetilde{M}\left(W_{f}\right) \in\{(0, \infty), \mathbb{R} \backslash\{0\}\} .
$$

Since (11) holds, we can define a nontrivial linear functional $h: X \rightarrow \mathbb{R}$ as follows:

$$
h\left(a x_{0}+y\right)=a \quad \text { for every } a \in \mathbb{R} \text { and } y \in A_{f} .
$$

According to (10) and (14), we have

$$
h(x)=\widetilde{M}(f(x))-1 \quad \text { for each } x \in F_{f} .
$$

Putting $x=0$ in (1), we get $f(M(1) y)=f(y)$ for each $y \in X$. Thus, (15) implies that

$$
h(y)+1=\widetilde{M}(f(y))=\widetilde{M}(f(M(1) y))=h(M(1) y)+1=M(1) h(y)+1
$$

for each $y \in F_{f}$. By (13) and (15) $h\left(y_{0}\right) \neq 0$ for some $y_{0} \in F_{f}$. Hence $M(1)=1$ and consequently, in view of (5), $\widetilde{M}=M$.

Moreover, by (11) and (12), we obtain that

$$
X \backslash F_{f}= \begin{cases}-x_{0}+A_{f}, & \text { if } M\left(W_{f}\right)=\mathbb{R} \backslash\{0\}, \\ (-\infty,-1] x_{0}+A_{f}, & \text { if } M\left(W_{f}\right)=(0, \infty) .\end{cases}
$$

Hence, in view of (14),

$$
h\left(X \backslash F_{f}\right)= \begin{cases}\{-1\}, & \text { if } M\left(W_{f}\right)=\mathbb{R} \backslash\{0\}, \\ (-\infty,-1], & \text { if } M\left(W_{f}\right)=(0, \infty) .\end{cases}
$$

On the other hand, using Lemma 2(ii), we obtain $M(f(x))=0$ for each $x \in X \backslash F_{f}$. Thus, according to (15), either

$$
M(f(x))=h(x)+1 \quad \text { for each } x \in X
$$

or

$$
M(f(x))=\max \{0, h(x)+1\} \quad \text { for each } x \in X .
$$

Hence $\left.M\right|_{f(X)}$ is injective and multiplicative.

Let $Z=M(f(X))$. According to (16) and (17) $Z \in\{[0, \infty), \mathbb{R}\}$. Let $H$ : $Z \rightarrow \mathbb{R}$ be given as follows:

$$
H(z)=\left(\left.M\right|_{f(X)}\right)^{-1}(z) \quad \text { for } z \in Z .
$$

Function $H$ is multiplicative and injective on $Z$. We prove that $H$ is continuous on $Z \backslash\{0\}$.

To this end fix $y \in X \backslash$ ker $h$. According to Lemma 9 there are $z \in F_{f}$ and a set $B \subset \mathbb{R}$ with $m(B)>0$ such that $B \subset f_{y}^{-1}\left(f(z)^{-1} f(D)\right)$. If $f$ and $M$ satisfy (17), then $h(B y)+1 \subset(0, \infty)$ (otherwise we would have

$$
0 \in\{\max \{0, h(x)+1\}: x \in B y\}
$$

and hence

$$
0=H(0) \in H(\{\max \{0, h(x)+1\}: x \in B y\})=f(B y) \subset f(z)^{-1} f(D) \subset W_{f},
$$


which is a contradiction). Thus $H(h(B y)+1)=f(B y)$ and, by the linearity of $h$,

$$
|H(B h(y)+1)|=|f(B y)| \subset\left|f(z)^{-1} f(D)\right| \subset\left(0, a_{0}\right)
$$

for $a_{0}=|f(z)|^{-1} a$. Since $m(B h(y)+1)>0$, we conclude that $H$ is a multiplicative function bounded on a set of positive Lebesgue measure. Thus, according to $[1$, Corollaries $7-8$, p. 31] $H$ is continuous on $Z \backslash\{0\}$. Therefore, as $H$ is injective, applying [1, Corollary 9, p. 31], we get that $H$ is given by

$$
H(z)= \begin{cases}|z|^{c} \operatorname{sgn} z, & z \in Z \backslash\{0\} ; \\ 0, & z=0\end{cases}
$$

with a certain $c \in \mathbb{R} \backslash\{0\}$. Then, by (16), (17) and (18), condition (iii) is valid. This ends the first part of the proof.

The converse statement is easy to check.

Remark 1 . If we additionally assume in Theorem 1 that $M \circ f$ is bounded on $D$, then the proof of this theorem can be simplified in a very natural way. First we have to prove the multiplicativity and the injectivity of $M$ on $f(X)$ (in exactly the same way as in the proof of Theorem 1). Then a function $\widetilde{f}: X \rightarrow \mathbb{R}$ given by $\tilde{f}=M \circ f$ satisfies (3) and, by Lemma $3(\mathrm{ii}),|\tilde{f}(D)| \subset\left(0, a_{0}\right)$ for some $a_{0}>0$. Therefore, to finish the proof we can apply [20, Theorem 1] (with $n=1)$.

The above-mentioned method potentially may not be applicable.

Remark 2. Note that in Theorem 1(iii) if $c>0$, then $M$ and $f$ are both continuous.

Indeed, if $(8)$ holds, then $h(D)+1 \subset(0, \infty)$ (otherwise $0 \in f(D)$, which contradicts the assumption). Thus, for $f$ given by $(7)$ or (8), we have

$$
h(x)=f(x)^{\frac{1}{c}}-1 \quad \text { for } x \in D .
$$

Hence the boundedness of $f$ on $D$ implies the boundedness of $h$ on $D$. Thus, in view of $[1$, Theorem 8, p. 17], $h$ is continuous and, by (7) or (8), so is $f$.

Open Access. This article is distributed under the terms of the Creative Commons Attribution Noncommercial License which permits any noncommercial use, distribution, and reproduction in any medium, provided the original author(s) and source are credited.

\section{References}

[1] Aczél, J., Dhombres, J.: Functional equations in several variables. In: Encyclopedia of Mathematics and its Applications vol. 31. Cambridge University Press, UK (1989)

[2] Beck, A., Corson, H.H., Simon, A.B.: The interior points of the product of two subsets of a locally compact group. Proc. Am. Math. Soc. 9, 648-652 (1958) 
[3] Brzdȩk, J.: Subgroups of the group $Z_{n}$ and a generalization of the Gołąb-Schinzel functional equation. Aequationes Math. 43, 59-71 (1992)

[4] Brzdȩk, J.: The Christensen measurable solutions of a generalization of the GołąbSchinzel functional equation. Ann. Polon. Math. 64(3), 195-205 (1996)

[5] Brzdȩk, J.: Bounded solutions of the Gołąb-Schinzel equation. Aequationes Math. 59, 248-254 (2000)

[6] Brzdȩk, J.: Gołąb-Schinzel equation and its generalizations. Aequationes Math. 70, 1424 (2005)

[7] Brzdȩk, J.: On the quotient stability of a family of functional equations. Nonlinear Anal. 71, 4396-4404 (2009)

[8] Christensen, J.P.R.: On sets of Haar measure zero in abelian Polish groups. Israel J. Math. 13, 255-260 (1972)

[9] Christensen, J.P.R.: Topology and Borel Structure. North-Holland, Amsterdam; American Elsevier, New York (1974)

[10] Chudziak, J.: Approximate solutions of the genearalized Gołąb-Schinzel functional equation. J. Approx. Theory 136, 21-25 (2005)

[11] Chudziak, J.: On a functional inequality related to the stability problem for the GoląbSchinzel equation. Publ. Math. Debrecen 67, 199-208 (2005)

[12] Chudziak, J.: Stability of the generalized Gołąb-Schinzel equation. Acta Math. Hung. 113, 133-144 (2006)

[13] Chudziak, J.: Stability problem for the Gołąb-Schinzel type functional equations. J. Math. Anal. Appl. 339, 454-460 (2008)

[14] Chudziak, J., Tabor, J.: On the stability of the Gołąb-Schinzel functional equation. J. Math. Anal. Appl. 302, 196-200 (2005)

[15] Fischer, P., Słodkowski, Z.: Christensen zero sets and measurable convex functions. Proc. Am. Math. Soc. 79, 449-453 (1980)

[16] Gołąb, S., Schinzel, A.: Sur l'équation fonctionnelle $f(x+f(x) y)=f(x) f(y)$. Publ. Math. Debrecen 6, 113-125 (1959)

[17] Jabłońska, E.: On solutions of a generalization of the Gołąb-Schinzel equation. Aequationes Math. 71, 269-279 (2006)

[18] Jabłońska, E.: Continuity of Lebesgue measurable solutions of a generalized GołąbSchinzel equation. Demonstratio Math. 39(1), 91-96 (2006)

[19] Jabłońska, E.: Bounded solutions of a generalized Gołąb-Schinzel equation. Demonstratio Math. 42(3), 533-547 (2009)

[20] Jabłońska, E.: Solutions of some functional equation bounded on nonzero Christensen measurable sets. Acta Math. Hung. 125(1-2), 113-119 (2009)

[21] Jabłońska, E.: Jensen convex functions bounded above on nonzero Christensen measurable sets. Ann. Math. Sil. 23, 53-55 (2009)

[22] Jabłońska, E.: Christensen measurable solutions of some functional equation. Nonlinear Anal. 75(2), 2465-2473 (2010)

[23] Jabłońska, E.: Solutions of a Gołąb-Schinzel-type functional equation bounded on "big" sets in an abstract sense. Bull. Aust. Math. Soc. 81, 430-441 (2010)

Eliza Jabłońska

Department of Mathematics

Rzeszów University of Technology

W. Pola 2, 35-959 Rzeszów

Poland

e-mail: elizapie@prz.edu.pl

Received: April 21, 2010

Revised: October 4, 2010 